\title{
Interactive comment on "A water risk index for portfolio exposure to climatic extremes: conceptualization and an application to the mining industry" by Luc Bonnafous et al.
}

\section{Z. Wang (Referee) \\ w.zifeng@unesco.org}

Received and published: 7 December 2016

The authors' work is relevant to the emerging socio-hydrology community and meets the scope and focus of the journal. Global water risks that manifest in financial sector remain a critical concern, this article have made lucid but effective efforts to address such challenges from a perspective of Sovereign Wealth Funds on the specific topic of extreme precipitation induced losses for one or a portfolio of mines. Although corresponding hazards, exposures and risks have been well studied for a long time particularly by insurance and reinsurance companies, the water risk at portfolio level implying inherent spatio-temporal correlation (from ENSO e.g.) has never seen quantified for comparison, at least not publicly. 
I particularly like 2 points in the article: 1) The methodology of developing a global water risk index from long-term reanalysis datasets. I have seen many studies on the development of global water-related indicators, while how the index developed in this article is insightful (part 2 "structuring a risk index for climate extremes"). It provides a concise view on massive globally-historical data. 2) applying general extreme value distribution to precipitation time series makes the exceedance probability much more realistic than using empirical distribution (P18 -22 , "evidence of very fat tails for the portfolio risk"). I believe this application and fat tails finding have substantial implication for portfolio management. However, I would point out some confused points need to be better clarified and explained to hopefully improve the paper in some respects.

The major damages for a mine as mentioned in part 2 are spills and tailings dam. Precipitation is only one factor among all related climate or hydrological factors leading to those damages e.g. soil texture, runoff generation, groundwater level etc. It is much better but indeed very hard if not impossible to consider many involved factors both from data side and from physical mechanism side. It is also not conclusive to tell which is more informative when selecting one or several best variables. In spite of those, the combination of extreme precipitation and the capacity of runoff generation is usually regarded as appropriate balance of comprehension and feasibility in general situation. Therefore, I would suggest the authors expand the discussion of devising risk index in part 2 from more hydrological perspectives to better explained the reason selecting precipitation only. Integrating more hydrological variables with precipitation could be one improvement in the future.

The relation between "climate exposure" and "financial risk" is the central of the research and is built up on estimation of two variables, i.e. exceedance risk (eq. 1)

$$
n_{i, t}^{p}
$$

Printer-friendly version

and potential loss (eq. 3) 
A basic question raised here is to what extent eq. 1 and corresponding stationary and non-stationary threshold selection would represent "climate exposure"? It probably be more convincing if calibration or(and) validation is included in Example application parts, or mines in Queensland, South Africa introduced in part 1 are used. And same question to eq. 3 with regard to production loss assumptions (12.5\%, 11\%).

As far as I understand, eq.5 and eq. 6 are absolute and relative value of the same concept. So accordingly the difference between Table 1 and Table 2 is a multiplier, the value of the mines those companies hold. Based on above, I do not fully understand para. 1 in page 25 on the analysis of implications from different ranking, e.g. how "geographical variability" or "discrepancy" is associated with different ranking.

In general, I think this article is an interesting and meaningful work having connected water risk and real world loss and the methodology proposed has potential to be practical framework for water risk managers by adding their insider information. Besides, some minor comments and technical corrections:

1) P4, L19: "300 hundred houses" $\rightarrow$ "300 houses"? please re-check the number;

2) P6, L23: Is "web based application" available? If so, better to add a link in the article;

3) P11, L20: please specify full name of "NAV" at first mention;

4) Fig. 3 - 8: not as sharp as those in Appendices, re-print of high-res probably needed;

Interactive comment on Hydrol. Earth Syst. Sci. Discuss., doi:10.5194/hess-2016-515, 2016. 